\title{
Appunti di un professore di storia contemporanea. I dilemmi che l'insegnamento pone alla disciplina
}

\section{Mariuccia Salvati}

Storicamente, 1 (2005).

ISSN: 1825-411X. Art. no. 64. DOI: 10.12977/stor525

\section{La domanda degli studenti:}

a) Exit, ovvero scegliere con i piedi

E' difficile fare un calcolo, almeno per ora, dei flussi degli studenti fra i vari corsi di laurea delle facoltà umanistiche e in particolare fra quelli che fanno capo alla Facoltà di Lettere e Filosofia di Bologna. Troppe le novità che la recente istituzione del $3+2$ ha comportato, ancora difficilmente valutabili. Tuttavia, alcune cose si notano: nella tripartizione canonica dell'età postantica, il flusso degli studenti tende a privilegiare l'età contemporanea, segue quella medievale e buon ultima l'età moderna: se interrogati, gli studenti di primo anno rispondono che questa appare loro come una sorta di età di mezzo priva di 'identità'. Inoltre, in maniera imprevista, conosce un successo notevole il corso in Scienze antropologiche e religiose, ha un buon numero di iscritti il nuovo corso di Geografia, aumentano gli studenti del corso di laurea Orientale, mentre mantiene la sua inesauribile attrattiva il corso in Scienze della comunicazione.

b) una tesi di laurea triennale del 2005

Globalizzazione e rinascita del centro storico a Mercato Saraceno : è il titolo di un elaborato in un certo senso 'esemplare'per il nostro argomento, da più 
punti di vista. Preciso che l'argomento iniziale è stato suggerito dalla studentessa (Chiara Magnani), iscritta al corso triennale di laurea in storia. E' di circa 100 pagine, corredato di mappe e fotografie, fonti e bibliografia (contro ogni idea 'facile' della tesi triennale...). La prima parte, Decadenza e rinascita urbanistica, descrive gli attori sociali, politici e istituzionali che si sono confrontati sul territorio a partire dal 1971, anno terminale, nella storia della cittadina, di un declino demografico durato per vent'anni, durante i quali si era assistito al trasferimento della popolazione attiva nei centri della pianura cesenate o addirittura nel triangolo industriale del nord-ovest. La svolta interviene, appunto, negli anni '70 con la rivitalizzazione degli organi del decentramento amministrativo e con la costruzione della superstrada Orte -Ravenna. Così, nei decenni '80-'90 tutto cambia, non più in ragione dei programmi dei partiti ma prevalentemente su input della Regione (Piano territoriale), della nuova provincia Forlì-Cesena e dell'UE ( Green Paper on the Urban

Environment

<http://europa.eu.int/comm/off/green/index_en.htm>). La seconda parte della tesi è dedicata alla rinascita del centro storico e al confluire sull'obiettivo di questa trasformazione di soggetti sia pubblici che privati in funzione di una ripresa del suo ruolo commerciale. Fondamentale, anche per questa piccola cittadina, è il ruolo della cultura, come spiega S. Vicari Haddok [1], a sostegno dello sviluppo del turismo ambientale. Uno dei punti forti in questo risultato è il ritorno della storia . Il cosiddetto "Palio del Saraceno" ne costituisce un esempio - con abitanti in maschera, corteo e cena medievale - che si ripete ogni anno a settembre. Il paradosso è che il Palio è una mera invenzione (il Saraceno del nome della cittadina ha poco a che fare con le disfide cavalleresche), che tutti lo sanno, ma che i cittadini partecipano con entusiasmo mentre i visitatori si affollano nei centri commerciali. Fame di storia, bisogno di comunità e buoni affari rappresentano una combinazione di sicuro successo e volentieri si è disposti a inventare o a mercificare la propria tradizione. Altra annotazione: tutte le fonti di questa tesi, comprese le leggi nazionali, i regolamenti europei, le statistiche economiche e la bibliografia sull'argomento, sono stati tratti da siti 
internet.

Tutto ciò pone nuovi interessanti interrogativi: l'età contemporanea risulta per il nuovo studente di storia essere legata a un contesto spaziale collocato in un continuum che va dal locale al globale saltando i confini nazionali, quale riflesso di profondi cambiamenti istituzionali: come nelle prime scene del film di W. Wenders, Lisbon Story (1995), dove l'amico fonico chiamato dal regista a Lisbona percorre il lungo tragitto - climatico, geografico, storico - che lo conduce da Francoforte verso la capitale portoghese senza incontrare barriere (siamo appena dopo Schengen e l'esperienza suscita stupore, gioia e...orgoglio), anche qui gli amministratori locali dialogano con la comunità europea molto di più che con il parlamento nazionale, mentre i cittadini fanno i conti con la fine di una industrializzazione da grandi fabbriche, il mercato locale scopre con gioia l'apertura europea e allo Stato chiede soprattutto un sostegno traducibile in strutture viarie e di comunicazione.

Paradossalmente, in questo nuovo contesto, la storia diventa non tanto un cemento identitario quanto un campo di invenzione funzionale al successo del mercato locale. II ricercatore di storia ritrova una propria collocazione, anche economica, come inventore di miti e di riti, relegando nel passato il ruolo di "costruttore dello spirito nazionale" che gli era stato assegnato negli ultimi due secoli. Possiamo dircelo: la novità è molto più radicale del boom dell' "effimero" che aveva visto gli assessori regionali alla cultura mobilitarsi negli anni '80 per catturare il consenso elettorale dei giovani. Oggi l'intreccio tra cultura e mercato - a differenza di quando, come vent'anni fa, gli sponsor erano le grandi industrie o il bilancio pubblico - alimenta un boom di servizi che si diparte dal locale per incontrare non la nazione ma l' Europa. La cultura che vi si esprime si basa su percorsi che collegano monumenti e siti cultural- religiosi del territorio europeo: il tour del barocco, quello dei castelli medievali, i giubilei, i pellegrinaggi (Santiago de Compostella, Via Francigena), per non parlare del turismo gastronomico, rafforzano apparentemente il protagonismo della storia ma il tutto è finalizzato a 
coinvolgere soggetti finanziari e mercantili extra-nazionali, quantomeno europei.

Persino gli archivi sembrano aver perso la loro funzione di strumentomonumento [2] della memoria storica nazionale. A chi importa ormai sapere se la Disfida fra i Castelli Saraceni con Torneo a Cavallo e Corteo Storico ha davvero a che fare, da un punto di vista filologico, con il passato medievale di Mercato Saraceno? In compenso, gli effetti del Green Paper della UE vengono studiati dai nostri studenti di storia contemporanea a Cork come a Mercato Saraceno [3]. E la cosa funziona! Perché tra le due aree (Irlanda e Romagna) e i recenti sviluppi del mercato globale si riscontrano analogie sorprendenti, frutto di cambiamenti tecnologici epocali recenti, che solo uno sguardo "innocente" riesce a scoprire.

Già, che cosa significa innocente in questo caso? Proprio quello che intendevano i situazionisti negli anni '60 quando parlavano dei muri "non innocenti" della Parigi che aveva conosciuto le ristrutturazioni di Haussman e i cortei della Comune del 1871, alla vigilia delle manifestazioni studentesche del '68. Parlare di innocenza in questo caso è un po' come evocare il nietzschiano "per ogni agire ci vuole oblio" [4]; significa lasciarsi alle spalle la difficile e conflittuale storia millenaria di tante località, per proiettare i nuovi 'siti' su un unico sfondo globale virtuale, senza remore e senza quel carico particolare che ci ha lasciato sulle spalle la storia dell'ultimo secolo. Sappiamo bene (noi, "volonterosi" cittadini europei) che ciò che scriveva Renan a proposito della moderna nazione francese («l'essenza di una nazione sta nel fatto che tutti i suoi individui condividano un patrimonio comune, ma anche nel fatto che tutti abbiano dimenticato molte altre cose» [5]), dovrebbe essere anche il nostro slogan per l'Europa: di quanti conflitti dobbiamo dimenticarci per poter aderire con convinzione a un progetto comune? Se ci siamo sempre dichiarati d'accordo con Renan per la formula della cittadinanza come "plebiscito di ogni giorno", ancor più dovremmo esserlo oggi sognando una cittadinanza europea; e chi meglio dei giovani storici è adatto a portare a compimento quell'oblio necessario? 
(«L'oblio, e dirò persino l'errore storico - ha scritto ancora Renan, cioè uno dei massimi filologi del suo tempo - costituiscono un fattore essenziale nella creazione di una nazione, ed è per questo motivo che il progresso degli studi storici rappresenta spesso un pericolo per le nazionalità» [6]). Chi meglio di un giovane storico può promuovere l'oblio di alcuni eventi storici? Infatti, per dimenticarli occorre prima conoscerli!

Colloco a questo punto anche un'altra osservazione paradossale, in vista delle nostre riflessioni sulle ricadute 'disciplinari': il parallelismo che accomuna il gesto del giovane che sceglie di studiare un soggetto dislocato nel tempo con quello che lo cerca nello spazio: già J. Revel, qualche anno $\mathrm{fa}$, in una lezione tenuta a Bologna, nella sede di S. Giovanni in Monte, aveva notato nel gusto del pubblico per il passato medievale (una vague che in Francia ha preceduto quella europea) un bisogno di "esotico" che noi ritroviamo analogo nella domanda dei giovani che si iscrivono a antropologia, storia orientale, geografia o storia contemporanea (indirizzo "mondo contemporaneo").

L'antropologo Marc Augé ci spiega che, mentre cresce la rapidità di comunicazione e aumenta il tempo passato nei non-luoghi (aeroporti, autostrade, centri commerciali), aumenta anche in proporzione il nostro desiderio di luoghi dotati invece di forte identità, nello spazio e nel tempo: centri commerciali ma anche Palio del Saraceno, aeroporto JFK di New York ma anche villaggio nel Chiapas... [7]

Tirando le fila da queste considerazioni: a me sembra che la pressione sui confini disciplinari della storia contemporanea si stia facendo troppo forte, rispetto anche solo a qualche anno fa, con rischi di incrinatura evidenti e senza che vi si presti una attenzione adeguata.

\section{Le risposte della disciplina M-STO/04}

La disciplina Storia contemporanea (corsi, manuali, curricula) ha conosciuto una indubbia apertura tematica e una nuova dimensione geografica dopo il 
1989. E' ancora il caso di notare che, in coincidenza con gli insuccessi della esportazione forzata di un astratto modello di modernizzazione, è cresciuto parallelamente il bisogno di storia proveniente dalle discipline a cui ci si era maggiormente appoggiati in passato, come l'economia e le scienze sociali (teorie di path dependance, institutional economics, historical institutionalism ). Sono peraltro note le vere e proprie rivoluzioni metodologiche che hanno investito la storia ma anche le scienze umane in genere, quali antropologia e geografia: ritorno dell'evento, cultural e linguistic turn, ermeneutica, decostruzione testuale ...

Tralasciando il campo della teoria della storiografia, a cui pure I'Italia ha offerto un importante e originale contributo, a partire dall'incontro tra storia sociale e microstoria, mi limiterei a ricordare alcuni passaggi del contesto nazionale e accademico-disciplinare. Dunque, schematicamente si può affermare che nel dibattito italiano sulla storia contemporanea (la prima cattedra è della metà degli anni 60) seguito al '68 e alla contestazione delle chiusure partitiche e/o dell'impianto ideologico- storicista che l'avevano contrassegnata nei decenni postbellici, le principali spinte innovative siano provenute da un lato dalla storia sociale [8] e dall'altro (ma i due fatti sono correlati) dalla riflessione sulle "vie particolari" della modernizzazione italiana rispetto agli altri grandi paesi industriali ai quali ci stavamo rapidamente avvicinando sul terreno economico. Di conseguenza, nel corso degli anni ' 70 la prospettiva comparata e l'approccio interdisciplinare ai processi di modernizzazione appaiono saldamente impiantati nella nostra contemporaneistica, proprio mentre nascono e si diffondono i corsi di laurea in storia.

Se posso permettermi una citazione personale, in una rassegna di 10 anni fa pubblicata in Francia, Storia contemporanea e analisi comparativa in Italia [9] potevo documentare il diffuso ricorso all'approccio comparato nella produzione storiografica recente a partire da alcune parolechiave, come neocorporatismo, fascismo, resistenza, borghesie, classi sociali, cittadinanza, welfare. Più in generale era anche possibile evidenziare quel tratto distintivo 
del comparatismo italiano che consiste nel sottolineare la anomalia del caso italiano; a mio avviso, cioè, lo sguardo comparato ha accompagnato quella sorta di costante controstoria nazionale imperniata su questione meridionale, trasformismo, fallimento delle classi dirigenti, ma ha anche consentito una originale e innovativa rivalutazione dei "territori" e della loro singolarità, lungo una pluralità di percorsi di modernizzazione sub-nazionale indagati al di fuori di ogni economicismo. Terreno di incontro disciplinare ideale è stato non a caso la einaudiana collana sulle Storie regionali [10].

Se questo è lo sfondo storiografico, quali sono i riflessi istituzionaldisciplinari?

a) La Sissco (Società Italiana per lo Studio della Storia Contemporanea $<$ http://www.sissco.it/>)

La prima idea di un'associazione di contemporaneisti nasce in occasione di un convegno comparato sulle istituzioni municipali in Europa nel 1989 a Trento [11]. La nascita dell'associazione è l'occasione per una riflessione sulla storia contemporanea e su ciò che ne costituisce la specificità. Interessante notare che l'accordo generalizzato sul rifiuto della logica dell'appartenenza partitica lascia poi spazio a interpretazioni diverse sui fondamenti e il territorio della disciplina: da parte di alcuni si insiste sul metodo (la comparazione e il rapporto con le scienze sociali), da parte di altri sugli obiettivi specifici di un'associazione di storici italiani («riprendere in maniera complessa la storia d'Italia», propone, per esempio, Galli della Loggia) o ancora il richiamo all'identità della contemporaneistica attraverso il confronto sulla periodizzazione o sul processo di modernizzazione. Nello Statuto le finalità della Sissco venivano così definite: " confrontare $i$ diversi interessi di ricerca con lo statuto scientifico della disciplina, i suoi confini, $i$ suoi rapporti con le scienze sociali e con le tematiche che nascono dal confronto con altre epoche e altri paesi ».

Di fatto e per fortuna si verificò una combinazione di metodi e obiettivi, passando nei convegni annuali successivi dal tema de La nazionalizzazione culturale degli italiani 
(1991) a Autorità e potere politico nella storia dell'Italia contemporanea (1992), Religione e modernità. Una prospettiva comparata per la storia dell'Italia contemporanea (1993), Le classi dirigenti nell'Italia contemporanea (1994), a Le leggi elettorali nella storia d'Italia (1995), a II secolo ambiguo. Le periodizzazioni nel secolo XX (1996), I Linguaggi della Nazione (1997), Rivoluzioni. Una discussione di fine Novecento (1998), Cittadinanza (1999), La democrazia nel Novecento (2000), Il mondo visto dall'Italia (2002). E' evidente il passaggio da una riflessione sull'identità nazionale in un prospettiva comparata ( Sonderweg e nation building ) a una prospettiva da parolechiave, prospettiva di per sé interdisciplinare e sovranazionale. Nel frattempo altre iniziative legate allo stato della ricerca e della didattica sia universitaria che scolastica venivano ad affiancarsi ai convegni nazionali, con appositi seminari e incontri di crescente richiamo soprattutto tra gli studiosi più giovani. Un ponte innovativo e di grande successo tra seminario scientifico e partecipazione attiva di giovani ricercatori è tuttavia quello che viene lanciato nel 2001 a Urbino con l'incontro sul tema La storia contemporanea in Italia oggi, organizzato per seminari tematici a sessioni parallele. II successo della formula che assumerà il titolo Cantieri di ricerca prosegue nel 2003 a Lecce e si ripeterà nel 2005 proprio qui a Bologna. Se si tiene conto che nel frattempo era nata anche la Società Italiana delle Storiche (<http://www.societadellestoriche.it/>), e che tra le due associazioni si produce una sinergia crescente soprattutto nelle fasce generazionali più giovani, ecco che il bilancio della associazione dei contemporaneisti allo stato attuale induce a una prima considerazione e cioè l'inclusione di un'area di ricercatori giovani, e precari dal punto di vista accademico, ma in stretto contatto con l'università per vie diverse (i dottorati), su un progetto prevalente di storia sociale dal basso. Da tutto ciò deriva certamente una forte spinta al rinnovamento di fonti e metodi.

b) Su alcune riviste di storia contemporanea

La più recente rivista italiana di studi di storia contemporanea ha come titolo «Mondo contemporaneo»: nell'editoriale del primo numero (2005) si 
assicura infatti una dimensione spaziale "planetaria", mondiale. E il tempo? La storia contemporanea, si scrive, si è sviluppata, "ma il suo ambito concettuale (...) resta comunque indefinito e complesso"; quanto al metodo, mentre si confermano i principi canonici dello storico, qualunque sia la sua specializzazione - confronto sulla periodizzazione, centralità della ricerca si elencano, tra i temi privilegiati, le identità, le trasformazioni della politica, la cultura di massa, problemi di secolarizzazione e nuovo ruolo della religione. La rivista apre opportunamente con un dibattito sulla nozione di contemporaneità a proposito della quale pubblica un intervento di René Remond in cui il grande storico sottolinea soprattutto il carattere provvisorio di un confine temporale che non cessa di spostarsi man mano che grandi eventi si producono. Osservazioni analoghe troviamo in un saggio di Giorgio Chittolini che lega questo confine all'avvento della modernità, e in un altro di Paolo Prodi che lo mette in rapporto con la parabola dello Stato nazione [12] - La tendenza francese, ricorda Remond, è quella a definire l'epoca contemporanea come storia del "tempo presente"; e da tutto ciò derivano, per la contemporaneistica, conseguenze importanti, come l'eccesso delle fonti, ma anche la necessità di fissare alcune date tornanti, di riflettere sul succedersi delle generazioni, sul ruolo della memoria e delle testimonianze.

Si tratta di un dibattito - quello sulla contemporaneità - estremamente interessante e che continuamente si rinnova [13], come giustamente osserva Remond, in rapporto ai grandi eventi. Ma quali sono questi grandi eventi comunemente accettati dalla comunità degli storici come date periodizzanti [14]? In che modo, per esempio, fenomeni come la globalizzazione o le date dell'11 settembre 2001 o della guerra in Irak (ben presenti nelle menti degli studenti, come abbiamo visto) costringono lo storico a un nuovo e forse ancor più radicale ripensamento rispetto a quello avviato dopo la data quasi ovvia del 1989? Oramai non conta in quale paese ci si collochi, con più o meno lunga tradizione di studi di paesi extraeuropei: che lo si voglia o no, globalizzazione e rivoluzione informatica ci costringono a ripensare la cronologia della contemporaneità proprio come conseguenza 
della radicale abolizione di quelle gerarchie spaziali che una volta erano definite dal diverso grado di 'modernità' [15].

Certo, ci si potrebbe accontentare, come contemporaneisti, degli spazi acquisiti. Infatti, neppure il "Journal of Contemporary History», classica rivista americana nata nel 1965, ha sentito la necessità di dovere modificare il proprio impianto negli ultimi anni: semmai è mutato l'accento o il metodo con cui si guarda ad alcuni eventi cardine del XX secolo, come il fascismo, il nazismo, le guerre (con uno spostamento verso la storia culturale e la memoria collettiva). Ma di ridefinire il proprio ambito la rivista non sente affatto il bisogno, anche perché, afferma uno dei suoi direttori [16], dagli anni del suo avvio pionieristico, la rivista di storia contemporanea, unitamente alla disciplina, si è ulteriormente specializzata e come tale è stata accettata accademicamente: dunque perché cambiare?

In realtà, il panorama è meno lineare di quanto il «Journal» lo presenti. Proprio negli stessi anni in cui la rivista americana afferma la sua scelta di continuità nasce negli Usa la world history con relativi corsi universitari e rivista (1990) [17], per non parlare della crescita degli studi culturali, e dell'uscita di un'apposita rivista («Studi Culturali») anche in Italia. E' evidente che queste ultimi filoni di studi insidiano il campo della storia contemporanea dentro i confini stessi della disciplina e non semplicemente affiancandosi ad essa. In un recente Companion to Western Historical Thought (Blackwell 2002), le sfide ai "confini del pensiero storico occidentale" venivano così elencate: Ia nuova world history, la postcolonial history, la multicultural history of the nations - più le nuove tecnologie e i media visivi [18].

Come ho accennato prima, I'Italia è un paese particolarmente predisposto a recepire queste aperture proprio perché, per tradizione di studi e di pensiero, non è mai stato interamente dominato da una lettura univoca del processo di modernizzazione: gli storici dell' IMES (Istituto Meridionale di Storia e Scienze Sociali<http://www.imes.it/>) e di «Meridiana», per esempio, hanno da tempo messo in discussione quel modello unico che era stato dominante 
nel mondo occidentale nei primi decenni del secondo dopoguerra. Questo ha reso più facile la comprensione e l'uso di approcci post colonial al nostro, diciamo così, 'oriente interno', cioè al Mezzogiorno. II ponte in questo caso è rappresentato da Antonio Gramsci il cui pensiero ispira, come è noto, la generazione più nota e influente degli intellettuali e studiosi critici del modello occidentale della modernità.

Si profila inoltre anche in Italia il passaggio dalla storia sociale scientifica (come la definisce G. Iggers [19]) al postmodernismo e al culturalismo. Più difficile per gli storici nazionali il passaggio agli studi globali comparati, alla entangled history, anche se la collaborazione con gli antropologi può rappresentare un buon punto di apertura [20].

c) La manualistica

Prenderei in considerazione dapprima l'interessante opera di G. Procacci, La memoria controversa [21], impressionante rassegna di uso pubblico della storia come legittimazione del ceto politico seguito ai crolli dell'89: un revisionismo ufficializzato, una strumentalizzazione della storia con impreviste ricadute, soprattutto per quanto riguarda il settore dell'Asia o del Medio Oriente, ma che si ritrova anche nel dibattito sul multiculturalismo in USA. Procacci mette in luce quale tratto maggiormente preoccupante, nella dinamica recente anche nazionale, non tanto il revisionismo quanto la chiusura e in molti casi la condanna stessa dell'approccio 'mondialista' ( $R$. Bottiglione ha criticato I"astratto cosmopolitismo'): una tendenza che accomuna paesi molto diversi e che si rivela particolarmente forte in quelli di recente formazione o di recente conquista democratica (dove più forte è sentita la "necessità di una storia patria"). Interessante la sottolineatura invece della storia russa come storia della continuità della Grande Russia, da Ivan il terribile a Putin [22]. Culto del passato remoto (Alessandro il Grande conteso alla Grecia ei manuali di storia della Macedonia) e silenzio su quello prossimo (il manuale serbo senza Milosevic o quelli greci senza la guerra civile, fino agli anni (80). Altra tendenza è quella di una identità 
nazionale schiacciata su quella religiosa: induismo (India), islamismo (Pakistan). Infine vi è già un accenno forte alla vague revisionista del Giappone (rappresentato dai suoi manuali come il vero vincitore della II guerra mondiale), nella prospettiva della costruzione di una Grande Asia e di una guerra di liberazione dal dominio straniero: francese (Vietnam), inglese (India), olandese (Indonesia), americano (Filippine). La questione nippocinese è diventata un caso diplomatico - lo notava già Procacci nel dicembre 2003 [23] - e oggi rischia di diventare militare, intrecciandosi con il tentativo del governo cinese di deviare sullo scontro con il Giappone il crescente malcontento sociale dei suoi lavoratori [24].

Nei migliori manuali italiani di storia degli ultimi cento anni sembra ormai recepito quel passaggio descritto, o auspicato, da C. Maier, in occasione della lezione al Mulino del 1999 [25]: a fine secolo e millennio - constatava lo storico di Harvard - la ricerca storica si è spostata, anzi si dovrebbe spostare, dalla prima parte del secolo, cioè dai totalitarismi europei (su cui si era prevalentemente soffermata fino agli anni (90), alla seconda metà del '900, sforzandosi dunque di comprendere le guerre, vecchie e nuove, sia dell'Europa balcanica e dell'Oriente che del Sud del mondo. E infatti, negli ultimi tempi si è accentuata la tendenza, nei manuali di storia contemporanea, a includere nuovi pezzi di storia del mondo, con un allargamento di dimensioni difficile da maneggiare, in tutti i sensi...

Il campo della disciplina si espande, ma che cosa tiene insieme le sue estensioni geografiche? Come leggere la storia del globo con nuove chiavi interpretative, come compete alla storia, ma evitando le semplificazioni pericolose e soprattutto il facile ricorso alle contrapposizioni radicali: non solo il ben noto "scontro di civiltà", ma anche globalizzazione vs. moltitudini, ricchi vs. poveri, Nord vs. Sud, ecc.? Come ritrovare la specificità della dimensione storica (il tempo) di fronte alla sfida rappresentata dalla globalizzazione (cioè dallo spazio)? [26]

Non mancano peraltro validi tentativi di sintesi. E su questo terreno si 
potrebbero affiancare tre Guide tra le più significative e originali all'età contemporanea: G. Barraclough (1964), E. Hobsbawm (1994) e M. Flores (2002) [27]. Tralasciando di tornare qui sul Secolo breve [28], vorrei mettere in luce come il passaggio del testimone sia soprattutto evidente tra Barraclough e Flores, tra l'asse interpretativo del "dwarfing of Europe" nello storico inglese e quello dell'Occidentalismo/liberalismo e i suoi nemici, nello storico italiano: li accomuna, come è evidente, soprattutto la prospettiva globale e di conseguenza uno sforzo di sintesi tra storia politico-culturale e geopolitica [29], un approccio che induce entrambi a un'attenzione limitata e calante all'Europa nel Novecento: per tutti e due gli autori, in fondo, all'Europa compete lo spazio che questo continente ha saputo conquistarsi nelle statistiche mondiali sulla popolazione, sul PIL, sul suo potere politico e militare... Cioè ben poca cosa.

\section{Il "canone" europeo}

In un suo recente libro, II sipario, M. Kundera riflettendo sul ruolo del romanzo e sulle difficoltà dell'Europa a pensare la propria letteratura come una unità storica osserva:

" Ci sono due contesti elementari nei quali è possibile collocare un'opera d'arte: la storia della sua nazione (chiamiamolo il piccolo contesto ) o la storia sopranazionale della sua arte (chiamiamolo il grande contesto). Siamo soliti considerare la musica, in maniera del tutto naturale, nell'ambito del grande contesto (...); un romanzo, invece, a causa del legame con la sua lingua, è studiato in tutte le università del mondo nell'ambito del piccolo contesto nazionale. L'Europa non è riuscita a pensare la propria letteratura come un'unità storica e non mi stancherò di ripetere che in questo consiste il suo irreparabile fallimento intellettuale (...)

Quel che ho appena detto è stato formulato per la prima volta da Goethe: "La letteratura nazionale non rappresenta più granché ai giorni nostri, stiamo entrando nell'era della letteratura mondiale e spetta a ciascuno di noi 
accelerare tale evoluzione”. E' questo se vogliamo il testamento di Goethe. Un altro testamento tradito.» [30]

Riprendendo il "testamento tradito" di Goethe, penso che sia nostro compito quello di spostare anche la storia, insieme all'arte e alla letteratura, dal piccolo al grande contesto, cioè di pensare "europeo", di enucleare come si fa in letteratura (e si noti che i più pressanti inviti all'intreccio culturale provengono da figure letterarie che impersonano nelle loro biografie e nella stessa lingua usata la vitalità dell'incrocio culturale) un "canone storico" europeo, nel quale inserire anche quello nazionale. Ed è anzi focalizzando la peculiare capacità (di matrice illuministica) della cultura europea di aprirsi alle altre culture [31] che anche la storia contemporanea può ritrovare la sua specifica dimensione temporale e avanzare nuove ipotesi interpretative.

Intanto, se proviamo a collocarci sulla scala di Odessa, come auspica Dan Diner nel suo Raccontare il Novecento, anche il bilancio del confronto politico e culturale tra Europa e resto del mondo appare subito più problematico e fecondo [32].

Non a caso, già i discorsi sul metodo, prima richiamati, degli anni '80 sembrano farsi obsoleti. Per esempio, il tema dell'identità nazionale ( nation building ) in chiave comparata, che tanta parte ha avuto nei primi anni della Sissco, sta per essere aggirato, diciamo così, su un duplice versante: da un lato, in ragione di una progressiva stanchezza per la rigidità stessa del comparatismo (che induce a ipostatizzare gli oggetti: il comparatismo, si osserva, costruisce in maniera artificiale le strutture nazionali, sopravvalutandole, non considerando le esperienze individuali) in favore di altre formule, come histoire croisée, transferts culturali; dall'altro per una prospettiva sempre più sopranazionale e europea.

Indicativo il ruolo di uno storico come $\mathrm{H}$. Kaelble [33], uno degli autori più prestigiosi della ricerca sulle borghesie europee promossa negli anni '80 dal Centro di storia comparata di Berlino e che oggi pubblica una nuova ricerca europea [34] ma in una chiave da histoire croisée, e con una diversa 
gamma di argomenti rispetto a 20 anni fa: non più le classi sociali, la famiglia, il welfare bensì i temi della comunicazione (dai media agli intellettuali), la memoria, le norme sociali, le donne, i gruppi etnici, i consumi, la religione. Anche la prospettiva metodologica è cambiata: dalla ricerca delle 'cause', che aveva accompagnato la prima fase della storia sociale (le strutture economiche, l'applicazione di teorie sociologiche, la relazione con la politica) a un approccio di tipo euristico, incluso il ricorso a nuove fonti: foto, film, quadri, diari, memorie. Oltre al metodo, è mutato il quadro geografico ( transnational ), così come la partnership con altre discipline privilegiate (in questo caso, etnologia, letteratura, filosofia, diritto).

II fatto è, ed è facile constatarlo in queste nuove ricerche sulla storia europea, che con il 1989 sono cambiate le sfide poste dalla realtà: la crisi industriale, il declino dell'importanza delle classi sociali, e dei sindacati, l'attacco al welfare, tutto questo sposta l'attenzione dai blocchi sociali agli individui, dalle classi alle famiglie, dalla propaganda al linguaggio. II tentativo di spiegare la storia europea in termini sociali è stato sfidato dall'evento 1989; per lo storico nascono nuovi compiti: il tema dell'esclusione sociale, delle crisi urbane, dell'immigrazione o, sul versante politico, la centralità della società civile, dell'opinione pubblica. Se si guarda alla produzione scientifica dei grandi storici delle borghesie degli anni ' 80 , è evidente lo spostamento in corso: problemi di Zivilgesellschaft, nuova enfasi sui legami sociali, passaggio dalla teoria di una modernizzazione unica a quella delle modernità "multiple", sono le novità più evidenti. [35]

Il progetto presentato poche settimane fa da una serie di istituti di ricerca di 10 paesi europei sul 'tempo presente' nel quadro dei programmi di finanziamento della UE ha come oggetto I tre dopoguerra nell'Europa del '900 (I, II guerra mondiale e 1989) e come metodo la 'europeizzazione' delle prospettive, cioè il superamento del paradigma nazionale, per incapsulare lo spettro delle diversità europee, est-ovest, nord-sud.

Anche qui: si tratta di segni e non necessariamente di proposte già condivise 
in vista della necessaria ridefinizione dello statuto della storia contemporanea. Tuttavia, è certo che solo a livello europeo può emergere un orizzonte interpretativo in grado di superare la frammentarietà e disomogeneità delle ricerche, e nello stesso tempo di costituire una risposta credibile alla sfida della globalizzazione nella storia contemporanea. Gli esempi non mancano, da parte di una generazione di storici della contemporaneità che assume esplicitamente, anche se in maniera critica, la dimensione della storia europea. E' il caso di Dark Continent ( Le ombre dell'Europa ) di M. Mazower [36]: per lo storico greco-inglese al centro del '900 vi è l'Europa e al cuore dell'Europa vi è la Germania sia nella prima metà del secolo (come promotrice del Nuovo Ordine europeo) che nella seconda, come motore del successo economico europeo. Qui Germania è sinonimo di Mitteleuropea, dunque racconto degli spostamenti di popolazioni e di indicibili sofferenze nella ricerca di nuovi confini. Oppure si veda History in Fragments di R. Vinen [37]: una storia sociale d'Europa, come il titolo è stato reso in italiano? Sì, se per sociale intendiamo i gruppi, le famiglie, gli individui, una storia d'Europa in cui dominano le biografie di profughi e esuli (e sarebbe da sottolineare anche il ritorno del genere biografico nelle simpatie di studenti e di giovani ricercatori): una histoire croisée vissuta, che ci restituisce una rappresentazione dell'Europa come "comunità immaginata" [38] prima di essere una comunità istituzionalizzata. Immaginata e costruita mentalmente, a prezzo di sofferenze, di guerre civili, di morti e che ha alla base un patto comune implicito: never again! . Non è sempre vero che la storia la scrivono i vincitori [39]: alla lunga la memoria delle vittime può prendersi una rivincita col racconto, il racconto aggiungerebbe Renan - di quanto, nel bene e nel male, è comunque 'nostro'. Del resto memoria e oblio non si elidono nella storia come in un gioco a somma zero...

Così l'Europa riacquista un suo protagonismo nella storia in virtù della memoria delle vittime, della memoria del 'sangue versato' [40], ma anche della sua rinascita morale e giuridico-istituzionale nel II dopoguerra [41]. 
Certo, si tratta di un protagonismo 'debole' ma non privo di una particolare attualità nel momento in cui nuove guerre e nuovi stermini nel mondo sembrano riproporre l'esigenza di una cultura dei diritti della persona conquistata, almeno per ora, da noi, cittadini europei, a così caro prezzo.

\section{Note}

[1]La città contemporanea, Bologna, Mulino, 2004.

[2] J. Le Goff, Documento/monumento , in Enciclopedia , V, Torino, Einaudi, 1978, 38-48.

[3] Mi riferisco a una tesi quadriennale ( Cork: spazio urbano e globalizzazione ), molto ricca e documentata, di Flavia Cumoli, sempre per Storia contemporanea (rel. Paolo Capuzzo).

[4] Sull'utilità e il danno della storia per la vita, Milano, Adelphi, 8; «E' proprio questa la considerazione a cui il lettore è invitato: ciò che è non storico e ciò che è storico sono ugualmente necessari per la salute di un individuo, di un popolo e di una civiltà », ivi, p.10.

[5] «Nessun cittadino francese sa se è Burgundo, Alano, Visigoto; ogni cittadino francese deve aver dimenticato la notte di San Bartolomeo, i massacri del XIII secolo nel Sud.» (E.Renan, Che cos'è una nazione, Roma, Donzelli, 1993, 8)

[6]lvi, p.7; e così prosegue: «La ricerca storica, infatti, riporta alla luce i fatti di violenza che hanno accompagnato l'origine di tutte le formazioni politiche, anche di quelle le cui conseguenze sono state benefiche; l'unità si realizza sempre in modo brutale; l'unificazione della Francia del Nord e del Sud è stata il risultato di uno sterminio e di un terrore durato ininterrottamente per quasi un secolo...»

[7] M. Augé, Nonluoghi: introduzione a una antropologia della surmodernità , Milano, Eléuthera, 1993; Id., Disneyland e altri non luoghi , Torino, Bollati 
Boringhieri, 1999.

[8] Cfr. A. M. Banti, Storie e microstorie: I'histoire sociale contemporaine en Italie (1972-1989) , "Genèses», 3 (1991),134-147 e G. Gribaudi, Premessa a «Quaderni storici», 94 (1997).

[9] «Genèses», 22 (1996), 146-159.

[10] Su questo tema e a partire da una introduzione di Lucio Gambi si è tenuto un seminario ( Attualità della Regione) presso la Fondazione Basso il 10 giugno 2003 di cui si dà notizia in "Le Carte e la Storia», 1 (2004). Si vedano il testo di Lucio Gambi e la relazione di Anna Treves in ACME, vol.VII, maggio-agosto 2004.

[11] II convegno (che poi diede luogo al volume F. Rugge (ed.), I regimi delle città . Il governo municipale in Europa tra '800 e '900, Milano, F. Angeli, 1992) era organizzato da F. Rugge, presenti M. Salvati e R. Romanelli. L'idea passa poi a Pisa e lì trova un terreno fertile nel gruppo dei contemporaneisti che fanno capo a L. Cafagna e C. Pavone: è su impulso di questo nucleo che si avviano le prime riunioni per costituire il comitato fondatore dell'associazione.

[12] G. Chittolini, Un paese Iontano , "Società e storia", 100-101 (2003), 331 ss.; P. Prodi, Storia moderna e storia contemporanea. II ruolo della storia moderna nella società italiana ed europea <http://www.giunta-storicanazionale.it/storici/forum.htm>.

[13] Si veda l'Annale IRSIFAR 1997 ( L'idea di contemporaneità e la trasmissione storica , Roma, Carocci, 1998) e ivi il mio L'idea di età contemporanea. Appunti per una ricerca .

[14] Per E. Hobsbawm ( II presente come storia, in Id. De historia , Milano, Rizzoli, 1997, 267) scrivere di età contemporanea comporta in primo luogo la selezione delle poche date storiche che costituiscono altrettante svolte per tutte le regioni del mondo e che formano la base di conoscenza comune per 
l'età contemporanea. A proposito di grandi date della storia colpisce il successo di una operazione curata da Alain Corbin ( 1515 et les grandes dates de l'Histoire de France revisitées par les grands historiens d'aujourd'hui , Paris, Seuil, 2005) a partire dalla cronologia di un noto manuale di storia per le elementari ( Cours élémentaire et moyen, di Blanchet e Toutin, ed. Belin, 1938) utilizzato in Francia negli anni '30 in una chiara chiave di 'costruzione della nazione' : 50 storici tra i più noti si misurano con 75 date considerate fondamentali nella storia del paese, pensando a un pubblico misto, composto di ex fruitori, di più giovani privi di riferimenti e di giovanissimi curiosi. Si tratta, ha osservato Corbin di "andare oltre il pubblico delle scienze umane perché ciascuno si renda conto che, al di là di un'ottica nazionale ormai obsoleta, la cronologia fornisce il senso di una architettura temporale, come una sorta di muro portante al quale appoggiare le date della altre civiltà, assenti o appena intraviste nel BlanchetToutain, ma di cui sappiamo di non poter più fare a meno oggi" (dall'intervista a Alain Corbin di Philippe-Jean Catinchi, «Le Monde», 28 gennaio 2005).

[15] Impossibile pretendere anche solo di citare qui il dibattito sui tempi della modernizzazione: è comunque un dato significativo delle svolte recenti di tipo "culturale" che il grande storico dell'economia D. Landes abbia impostato il suo recente affresco sull'avvento del capitalismo ( La ricchezza e la povertà delle nazioni: perché alcune sono così ricche e altre così povere , Milano, Garzanti, 2000; terza ed. ampliata 2002) a partire dall'intreccio tra geografia, economia e cultura. Ed è proprio la dimostrazione del ruolo svolto in economia dai processi storico-culturali e dai valori della libertà e della democrazia che ha portato alla ribalta un filone di economisti impegnati anche sul terreno di un più ampio confronto/incontro tra Occidente e Oriente (Amartya Sen)

[16] Arthur Marwick, A New Look, A New Departure: A Personale Comment on our Changed Appearance, gennaio 1997. 
[17] G. Gozzini lo segnala in "Contemporanea», 1/2004, un'altra rivista relativamente nuova del panorama italiano, nella quale peraltro è costante l'attenzione al dibattito internazionale: $v$. l'interessante rassegna di riviste storiche a cura di Neri Serneri, 4/2004, e l'ultima tavola rotonda su Traiettorie della 'World History' a cura di P. Capuzzo e E. Vezzosi, 1/2005.

[18] L. Kramer, S. Maza (eds.), A Companion to Western Historical Thought , Blackwell, 2002.

[19] G. Iggers ha tenuto, il 23 marzo 2005, un interessante seminario a Bologna nell'ambito del dottorato in "Storia d'Europa" (su invito di llaria Porciani, che lo ha intervistato per «Passato e Presente», 64 (2005)), sulle nuove tendenze della storiografia e il progetto di un approccio globale al processo della modernizzazione, nel tentativo di uscire da una prospettiva esclusivamente 'occidentale'.

[20] Si veda il n. 31 ( Occidentalismi ) di «Parolechiave» e la meritoria opera di traduzione di storici e teorici post-colonial da parte della casa editrice Meltemi.

[21] Cagliari, AM\&D, 2003, ma si veda anche la recente edizione Carte di identità. Revisionismi, nazionalismi e fondamentalismi nei manuali di storia, Roma, Carocci, 2005.

[22] Ma sulla chiusura all'occidente della Russia, cfr. il saggio di Maria Ferretti in Occidentalismi cit.

[23] G. Procacci , I/ dibattito internazionale sui manuali di storia, relazione al convegno Insegnare la storia in un mondo globale, Roma, Fondazione Gramsci, 11-12 dicembre 2003.

[24] Cfr. F. Rampini, «La Repubblica», 16 e 17 aprile 2005, dove si descrive con preoccupazione l'assenza di cultura storica nella formazione dei giovani giapponesi.

[25] Cfr. C.S. Maier, II Ventesimo secolo è stato peggiore degli altri? Un bilancio storico alla fine del Novecento 
, lettura dell' Associazione II Mulino, 13 novembre 1999, poi in «ll Mulino», 1999/6, 995-1011.

[26]A questo proposito mi permetto di riportare una considerazione storiografica avanzata nel mio II Novecento. Interpretazioni e bilanci, RomaBari, Laterza, 1999, 47: “E' interessante ancora segnalare come questo stesso fenomeno di "mondializzazione" (per usare il neologismo francese) si intrecci con una strutturazione, più o meno voluta, di tipo regionale che valorizza i fattori di "localizzazione". Questo "ritorno della geografia" come principio strutturante dell'organizzazione delle attività economiche (attraverso nuovi découpages incentrati sulle grandi aree metropolitane), favorito dalla fine della guerra fredda e dall'apertura delle frontiere, viene a sovrapporsi alle partizioni tradizionali di origine statale, che peraltro contribuisce a destabilizzare. In un confronto ideale con l'inizio del Novecento, si potrebbe quasi sostenere che alle soglie del nuovo secolo stiamo assistendo a una passaggio di testimone tra il tempo e lo spazio, tra la storia e la geografia ».

[27] G. Barraclough, An Introduction to Contemporary History, London, C.A. Watts \& Co. Ltd., 1964; E. Hobsbawm, The Age of Extremes: The Short Twentieth Century, London, Michael Joseph, 1994; M. Flores, II secolomondo. Storia del Novecento, Bologna, II Mulino, 2002.

[28] Su cui rinvio nuovamente a I/ Novecento cit.

[29] Naturalmente, nei circa 40 anni che separano i due libri è mutato soprattutto il rapporto tra passato e futuro. Tuttavia già in Barraclough come ora in Flores il primato dell'Occidente appare sotto forte pressione da parte dell'Oriente. Semmai nella sintesi più recente cambia la periodizzazione, con l'aggiunta della nuova svolta segnata dalla rivoluzione tecnologica che appiattisce la distanza temporale tra le diverse forme, ormai contemporanee, della modernità e colloca il passato novecentesco in un'unica epoca dominata dal sistema fordista. 
[30] Trad. it. Milano, Adelphi, 2005.

[31] Significativo di un incontro oriente/occidente imperniato su cosmopolitismo, umanesimo illuminista, amore per la musica e un'idea "fluida" dell'identità, il dialogo, durato per diversi anni, tra due figure di nomadi della cultura come Daniel Barenboim e Edward Said, ora raccolto in Paralleli e paradossi. Pensieri sulla musica, la politica e la società , a cura di Ara Guzelimian, Milano, II Saggiatore 2005.

[32] D. Diner, Das Jahrhundert verstehen : eine universalhistorische Deutung , München, Luchterhand, 1999; trad. it. Raccontare il Novecento: una storia politica, Milano, Garzanti 2001.

[33] Si fa qui riferimento a un recente seminario tenuto dallo storico di Berlino nella sede del Dipartimento di Discipline Storiche, su invito di Maria Malatesta , nell'ambito della scuola di dottorato in "Storia d'Europa", il 3 febbraio 2005.

[34] H. Kaelble (ed.), The European Way. European Societies in the 19th and 20th Centuries, London/New York, Berghahn, 2004.

[35]J. Kocka - Multiple Modernities and Negotiated Universal , in D.Sachsenmaier, J.Riedl with S.Eisenstadt (eds.), Reflections on multiple modernities. European, Chinese and Other Interpretations, Brill, Leiden, 2002 - riprende da Eisenstadt il concetto di "modernità multiple" spiegando che la globalizzazione crescente implica una interconnessione ma non necessariamente anche una omogeneizzazione crescente. Nel frattempo la definizione di modernità si fa più debole e il denominatore comune, tra le diverse forme di modernità, più sottile. Kocka avanza anche l'idea di "universali negoziati", proprio per la capacità dei concetti dell'illuminismo, a differenza del credo fondamentalista, di farsi modificare, aggiustare, "ibridare".

[36] M. Mazower, Dark continent : Europe's twentieth century, London, Allen Lane Penguin books, 1998. Trad. it.: Le ombre dell'Europa, Milano, 
Garzanti, 2000.

[37] R. Vinen, A History in Fragments. Europe in the Twentieth Century, London, Little, Brown, 2000. Trad. it.: L'Europa nel Novecento: una storia sociale, Roma, Carocci 2004.

[38] II riferimento è a B. Anderson, Comunità immaginate, Roma, Manifestolibri, 1996, ma soprattutto a C. Pavone, La seconda guerra mondiale: una guerra civile europea? , in G. Ranzato (ed.), Guerre fratricide. Le guerre civili in età contemporanea, Torino, Bollati Boringhieri, 1994.

[39] "Nel breve termine forse la storia è fatta dai vincitori; nel lungo termine le conquiste sul fronte della comprensione storica sono venute dagli sconfitti" (E. Hobsbawm, II presente come storia cit. ).

[40] Mi permetto di rinviare al mio II futuro dell'Europa tra globalizzazione e nazionalismi , «Parolechiave», 25 (2001), 101-114.

[41] P. Costa, Civitas. Storia della cittadinanza in Europa. 4. L'età dei totalitarismi e della democrazia, Roma-Bari, Laterza, 2001. 